\title{
Investigation of superlattice structure parameters using quasi-forbidden reflections
}

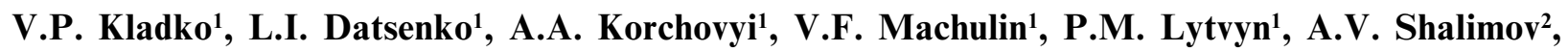 \\ A.V. Kuchuk ${ }^{1}$, P.P. Kogutyuk ${ }^{1}$ \\ ${ }^{1}$ V. Lashkaryov Institute of Semiconductor Physics, NAS of Ukraine, 45 prospect Nauky, 03028 Kyiv, Ukraine \\ E-mail:kladko@isp.kiev.ua \\ ${ }^{2}$ Institute of Physics PAN, 32/36 al. Lotnikow, 02-609 Warsaw, Poland
}

\begin{abstract}
We studied possibilities of a nondestructive X-ray technique for testing short-period strained GaAs-AlAs superlattices. An analysis of the quasi-forbidden 200 reflections may be used for determination of superlattice layer structure parameters and sublayer thickness. The effect of irregularity of superlattice transition region on X-ray diffraction reflection curves and elastic strains in layers was studied.
\end{abstract}

Keywords: X-ray structural analysis, superlattices, quasi-forbidden reflections.

Paper received 09.06.03; accepted for publication 17.06.03.

\section{Introduction}

X-ray diffraction (XRD) in structures where lattice period and diffraction maxima intensity are modulated along one of the coordinate axes (growth direction) is characterized by presence of satellites near the Bragg peak of the averaged crystal lattice [1,2]. The satellite peak intensities in a superlattice (SL) structures are proportional to the Fourier components of the modulated composition of the system studied. Besides, the changes of satellite peak widths in the reciprocal space carry useful information about perfection degree and coherence of SL layers [3,4]. Study of the so-called quasi-forbidden reflections (QFRs) permits perhaps to obtain new structure information. QFR was shown [5] to be very sensitive not only to structural defects but also to the lattice stoichiometry. So application of such reflections to investigation of periodic SLs when studying X-ray scattering by such structures seems to be promising and of great interest.

Our objective in this work was determination of layer parameters in GaAs/AlAs SL with X-ray diffraction (XRD) technique using QFRs.

\section{Theoretical and experimental procedures and subject of investigation}

When calculating X-ray diffraction patterns from an uniform epitaxial layer by the semikinematical theory [1], the following parameters are usually used: layer thickness $t$, polarizability (that is proportional to the structure factor $F_{h}$ ) and strain $\varepsilon$ in the SL structures. The total amplitude of X-ray scattering from a composite multilayer system may be described with a structural factor, that has the following form [3]:

$$
\begin{aligned}
& F(\vec{h})=F_{C a p}(\vec{h})+F_{M L}(\vec{h}) \cdot \exp \left(-i \vec{h} \cdot t_{C a p}\right)+ \\
& +F_{B}(\vec{h}) \cdot \exp \left(-i \vec{h} \cdot\left(t_{C a p}+t_{M L}\right)\right)+ \\
& +F_{S u b}(\vec{h}) \exp \left(-i \vec{h}\left(t_{C a p}+t_{M L}+t_{B u f}\right)\right)
\end{aligned}
$$

Here $t_{C a p}, t_{M L}$ and $t_{B}$ are the thicknesses of the upper and buffer layers of a SL, respectively; $h$ is the diffraction vector. The exponential factors in the expression (1) allow to describe the phase change of the scattering amplitude during X-rays propagation through the structure sublayers. When performing the corresponding calcula- 


\section{V.P. Kladko et al.: Investigation of superlattice structure ...}

tions the effect of layer thickness fluctuations $\Delta t_{i}$ was taken into account by the phase factor change:

$$
\varphi_{i}=\exp \left(-h^{2} \cdot\left(\Delta t_{i}\right)^{2}\right) .
$$

The SL studied here is a special case of an arbitrary layer structure. In the simplest case a SL period involves two layers (denoted further by $a$ and $b$ ). Each of them is characterized by its thickness, strain, structural factor and parameters $A$ and $Y$. The latter two are proportional to layer thicknesses and incident beam deviation from the exact Bragg angle. The diffraction amplitude for an $M$-periodic two-layer SL is of the following form [3]:

$$
\Phi_{M}=i \sqrt{\frac{\gamma_{0}}{\left|\gamma_{h}\right|}} \exp (-i \beta) F_{S} \frac{\sin \left[M\left(A_{a} Y_{a}+A_{b} Y_{b}\right)\right]}{\sin \left(A_{a} Y_{a}+A_{b} Y_{b}\right)}
$$

Here $\beta=(M-1)\left(A_{a} Y_{a}+A_{b} Y_{b}\right)+A_{a} Y_{a}$ stands for a phase factor, and structural factor, respectively, for one period is

$$
F_{S}=\frac{\sin \left(A_{a} Y_{a}\right)}{Y_{a}}+\exp \left[-i\left(A_{a} Y_{a}+A_{b} Y_{b}\right)\right] \frac{\sin \left(A_{b} Y_{b}\right)}{Y_{b}}
$$

The sine denominator in the expression (3) gives rise to peaks at

$A_{a} Y_{a}+A_{b} Y_{b}=n \pi$,

which are denoted as $n$-th order satellites $(n=0, \pm 1$, $\pm 2 \ldots)$. SL period can be determined from the angular spacing $\Delta \vartheta_{p}$ between these peaks:

$$
p=t_{a}+t_{b}=\frac{\lambda\left|\gamma_{h}\right|}{\Delta \vartheta_{p} \sin \left(2 \vartheta_{B}\right)} .
$$

The zeroth-order SL peak is off that of substrate one by the angle $\Delta \vartheta_{0}$, such that

$-\Delta \vartheta_{0}=\tan \vartheta_{B}\left\langle\varepsilon_{\perp}\right\rangle$

where $\left\langle\varepsilon_{\perp}=\Delta a / a\right\rangle$ is relative change of lattice period along the growth direction, and $\langle\ldots\rangle$ stands for averaging over this SL period.

Let us consider the simplest case of two-layer structure $(M=1)$. Then the expression for the structural factor becomes as follows:

$$
F_{S}=\frac{\sin \left(A_{a} Y_{a}\right)}{Y_{a}}+\frac{\sin \left(A_{b} Y_{b}\right)}{Y_{b}}\left(\cos \left(A_{a} Y_{a}+A_{b} Y_{b}\right)\right) \text {. }
$$

In the case of the GaAs two-layer system the structural factor for the QFR of 200-type (which is known to be proportional to difference between $\mathrm{Ga}$ and As atomic scattering factors) is rather small.

If one neglects the absorption and contribution from a substrate, then the expression for normalized intensity in structures with a center of symmetry may be written as following:

$$
\begin{aligned}
& R=|\Phi|^{2}= \\
& =\left(A_{1} \frac{\sin \left(A_{1} Y_{1}\right)}{A_{1} Y_{1}}+2 A_{2} \frac{\sin \left(A_{2} Y_{2}\right)}{A_{2} Y_{2}} \cos \left(A_{1} Y_{1}+A_{2} Y_{2}\right)\right)^{2}
\end{aligned}
$$

Taking into account the contribution to reflectivity from $\mathrm{GaAs}$ layer, one may obtain, after some simple mathematical manipulations:

$$
R=|\Phi|^{2} \cong 4 A_{2}^{2} \sin ^{2}\left(A_{2} Y_{2}\right) \cos ^{2}\left(A_{1} Y_{1}+A_{2} Y_{2}\right) .
$$

The rocking curves for the 400 and 200 reflections were taken using a triple-crystal X-ray diffractometer (Philips MRD, Institute of Physics PAN, Warsaw) equipped with the $4 \times \mathrm{Ge}(220)$ monochromator and $2 \times \mathrm{Ge}(220)$ analyzer. $\mathrm{CuK}_{\alpha}$ radiation was used. The samples studied were scanned near the Bragg angle over the $\sim 3^{\circ}$ range in the so-called $\omega / 2 \vartheta$-mode, as well as in the sample scanning mode. The measurements were carried out in the interval of $2^{\prime \prime}$. When analyzing the experimental data obtained, we used the traditional $\chi^{2}$-technique. It allowed to obtain both the above mentioned average parameters and their spread as well.

\section{Results and discussion}

Let us first consider the calculated rocking curves for a structure with a quantum well (Fig. 1). One can see from them that two diffraction pictures from regions differing in pendular oscillations periods may be recognized. The cosine term in the expressions (9) and (10) describes the total period of a binary layer, while the sine term depends on the AlAs layer thickness. A contribution from the GaAs layer is rather small because of small structural factor for the QFR 200. One can determine the total structure thickness as well as each layer thickness (from an simple rocking curve analysis). Based on the above

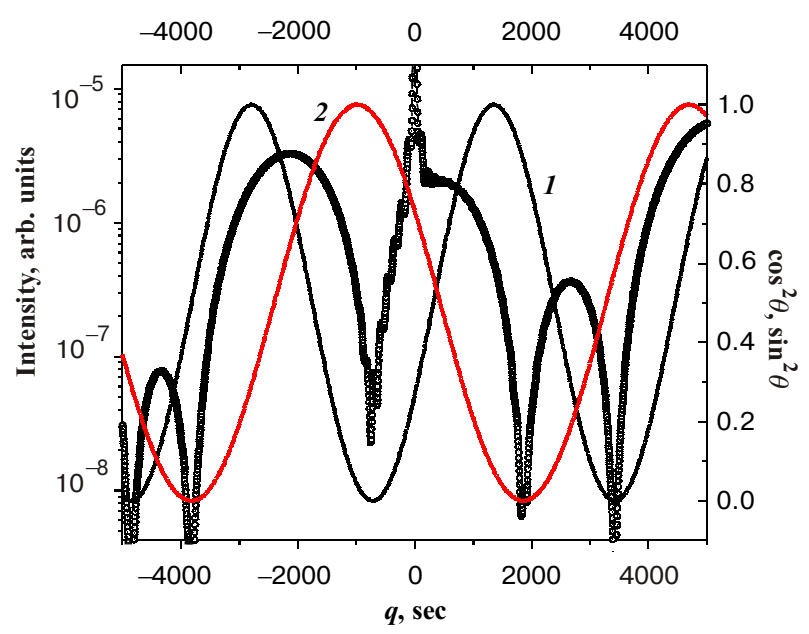

Fig. 1. Rocking curve for 200 reflex from GaAs-AlAs structure as well as behaviors of sinusoidal (I) and cosinusoidal (2) terms in expression (10). 
said, one may write down the following expression for the positions of two peaks:

$$
t_{1}+t_{2}=\frac{\lambda}{2\left(\sin \vartheta_{n+1}-\sin \vartheta_{n}\right)}
$$

Indices 1 and 2 denote GaAs and AlAs layers, respectively. The central peak of the sine function has also minima but with a period of $2 \pi$. From this one may obtain the $t_{2}$ value:

$$
t_{2}=\frac{\lambda}{\sin \vartheta_{2}-\sin \vartheta_{1}} \text {. }
$$

Using the expressions (6) and (7), one may obtain the strain value in the direction normal to the crystal surface $(\Delta a / a)_{2} \perp$, at the interface between two layers:

$$
\frac{t_{1}+t_{2}}{1+(\Delta a / a)_{m \perp}}=t_{1}+\frac{t_{2}}{1+(\Delta a / a)_{2 \perp}} \text {. }
$$

Here $(\Delta a / a)_{m \perp}$ is the calculated lattice mismatch for two layers in a free state. The experimental and calculated rocking curves for the GaAs(8 ML)/AlAs(4 ML) (ML here denotes a monolayer) sample studied using QFR 200 are presented in Fig. 2. One can see that both of the diffraction curves demonstrate fine spectral details, as well as satellite structure that is responsible for the value of SL period (Fig. 3). There is also a rather good agreement even between the fine structures of the experimental and theoretical rocking curves. It concerns the value of intensities near the zero-order satellite and the extremum positions as well.

Using a fitting procedure of the theoretical semikinematical curves to the experimental ones, some quantitative results concerning the SL parameters are possible to be obtained (by means of the above expressions). One may compare also these results with those derived from expressions (11)-(13). An analysis made shows that the data obtained by practically independent two approaches correlate rather well with each other. This fact indicates that our assumption concerning a contribution of the QFR 200 maxima into X-ray scattering is valid. The results of our measurements and calculations of the SL parameters are given in Table 1. Summing up all these results, one can state that the contribution from the layer 1 made up of components with close atomic numbers (GaAs) into the X-ray scattering intensity when using QFR is rather small. So, mainly the layer 2 consistence of components with considerably different atomic num-

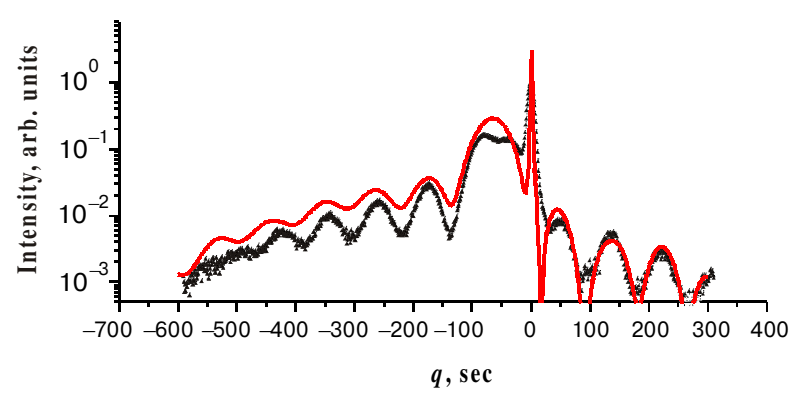

Fig. 2. Experimental (points) and simulated (solid line) rocking curves for QFR 200 ( $\omega$ scanning mode).

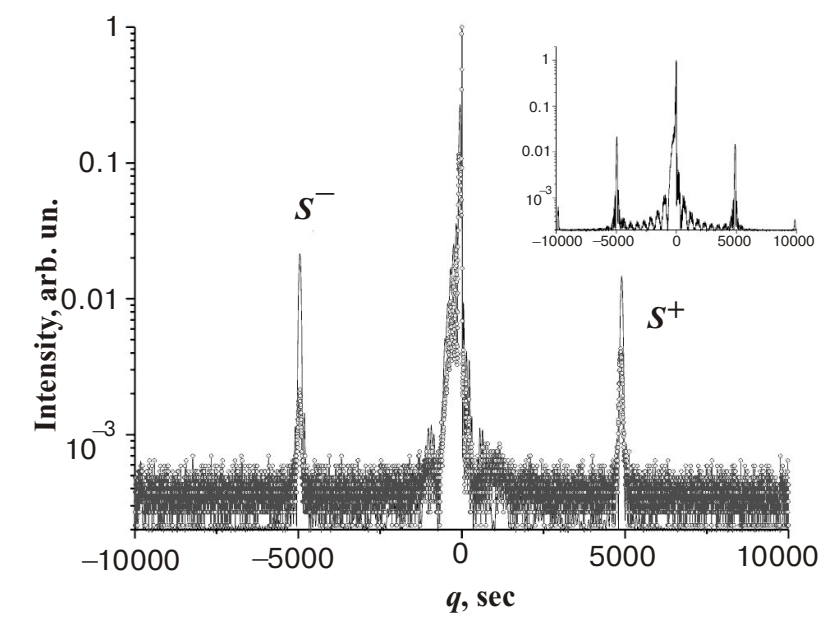

Fig. 3. Rocking curve for QFR 200 from SL: experimental (circles), simulated (solid line). Characters $\mathrm{S}^{+}, \mathrm{S}^{-}$denote first order satellites. On insertion the rocking curve simulated in consideration of layers thicknesses nonuniformity is shown.

bers determines the shape of 200 reflection. The main contribution from GaAs layer into total diffraction pictures is due just to phase factor changes. Nevertheless the satellite structure of these reflections is formed by both of layers. It is interesting to underline that the results of calculations show the general shape of rocking curves does not almost depend on crystal perfection of GaAs layer, i.e. on structure defects.

An interesting feature of QFR utilization consists in the rocking curve shape dependence on GaAs layer thickness. The results of the corresponding calculations are presented in Fig. 4. One can see that the character of

\begin{tabular}{|c|c|c|c|c|c|}
\hline $\begin{array}{l}\text { Sample } \\
\text { number/ } \\
\text { reflection }\end{array}$ & $\begin{array}{l}m \text { and } n \\
\text { sublayer } \\
\text { thicknesses, nm }\end{array}$ & $\begin{array}{l}\text { SL period } \\
T, \mathrm{~nm}\end{array}$ & $\begin{array}{l}\text { Calculated } \\
\text { strain for free } \\
\text { lattice }(\Delta a / a)_{m \perp}\end{array}$ & $\begin{array}{l}\text { Experimental data } \\
\text { according to } \\
\text { expression }(13),(\Delta a / a)_{m \perp}\end{array}$ & $\begin{array}{l}\text { Roughness } \\
\text { of SL sublayer } \\
\text { interface, nm }\end{array}$ \\
\hline НГ $8 / 4200$ & $22.48 \quad 11.07$ & 33.55 & 0.00132 & 0.0036 & 0.3 \\
\hline НГ $8 / 4400$ & 22.4511 .12 & 33.46 & & 0.0032 & 0.4 \\
\hline
\end{tabular}
intensity distribution near the satellite maxima changes

Table 1. The parameters of structures studied. 


\section{V.P. Kladko et al.: Investigation of superlattice structure ...}

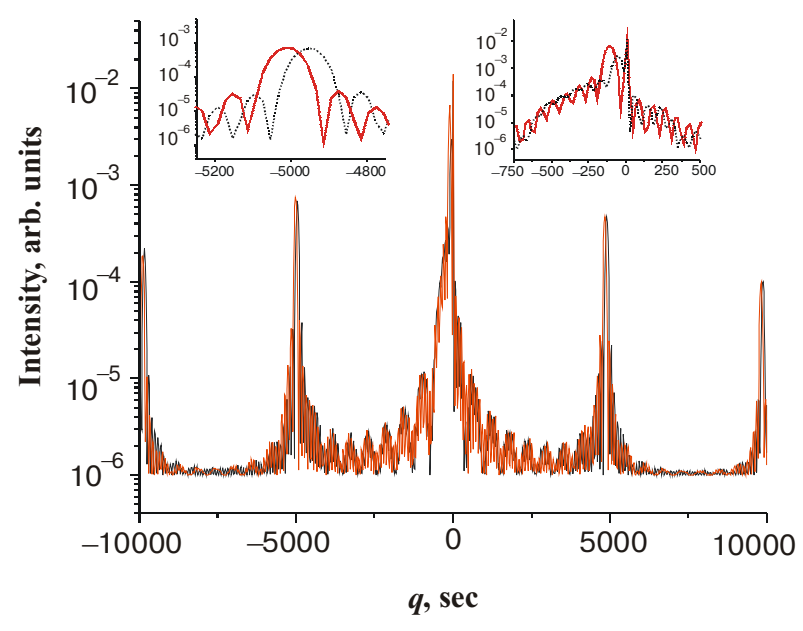

Fig. 4. The QFR 200 rocking curve shape dependence on thickness ratio of GaAs and AlAs layers: ratio 1:1 (dashed line), ratio 1:2 (solid line). On the insertions the enlarged scale for first order negative satellite (a) and zero order satellite (b) ranges are shown.

substantially when varying thickness of both (1 and 2) SL layers, by conservation of general SL period value.

Some words should be also said concerning the sensitivity of a satellite intensity to the layer thickness. For example, one should note a high sensitivity of scattering to layer thickness variations at the level of about $0.5 \mathrm{ML}$ in the case when thicknesses of both layers are close to each other. This result can be explained by an abrupt alteration of the phase factor at a slight variation of the thickness factor. Disappearance of satellites of a certain order can be also explained by the phase factor effect. When the real and imaginary parts of the corresponding SL structure factor for a satellite of a certain order are in antiphase, the satellites of this order disappear. These are the so-called "forbidden SL structural factors". Disappearance of satellites of a certain order depends on the relationship between the thickness of SL layer components. For example, if the layers have the same thicknesses, the satellites of the second order disappear. If the ratio of their thickness is equal, the maxima of the third order disappear.

At last, we have performed fitting the calculated rocking curves to the experimental ones. The results of this procedure showed that the layer surface roughness considerably affects diffraction pattern smearing, especially near the satellites of high orders. The first- and zerothorder satellites demonstrate low sensitivity of diffraction picture to the above structure imperfection parameter. Fig. 5 illustrates this. Our calculations are in good agreement with the experimental rocking curves (see Fig. 3). They demonstrate decrease of the second-order satellite intensity. The sizes of statistically distributed nonuni-

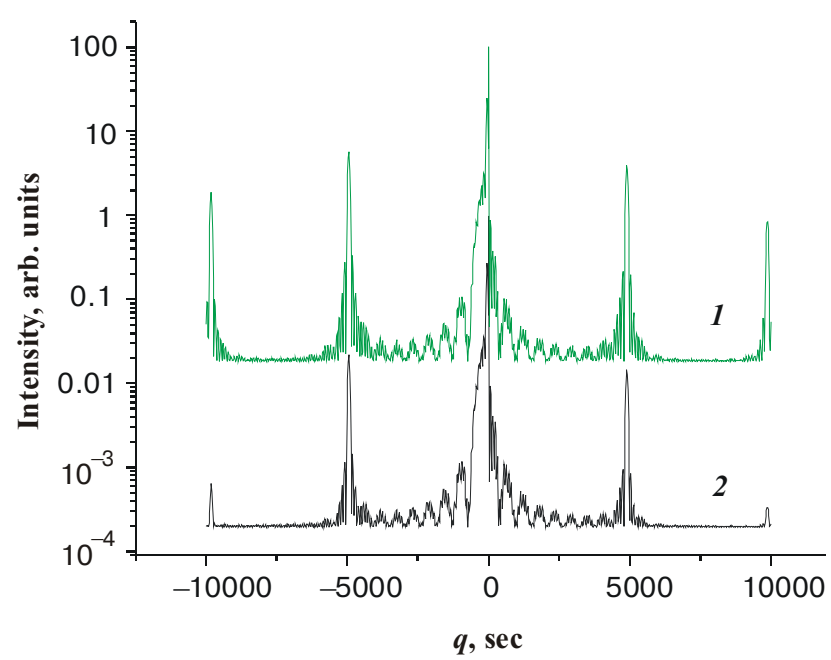

Fig. 5. The simulated rocking curves for the QRF 200 in the case of ideally smooth layers (I) and layers with roughness $0.3 \div 0.4 \mathrm{~nm}(2)$. formities of the interface between the SL layers occur in the $0.3-0.4 \mathrm{~nm}$ range. It should be also noted that asymmetry shape of the satellite peak intensities is observed in the case only when there are distortions of both types (variations of interplane spacing and scattering ability).

The calculation carried out showed the nonstoichiometry in the GaAs layer does not almost affect the general character of diffraction picture from SLs studied here. The level of the strain between two layers happened to be considerably higher as comparing with the value of mismatch $(\Delta a / a)_{m}$ between two lattices (GaAs and AlAs) (see Table 1).

\section{Conclusions}

Utilization of quasi-forbidden reflections when studying structural parameters of superlattices was shown to have several advantages over of the usual or structure reflections. First of all, a satisfactory agreement between the calculated and experimental rocking curves for quasiforbidden reflections was demonstrated. This indicates that semikinematical approximations provide a possibility for adequate description of the character of X-ray scattering. In the second place, use of quasi-forbidden reflections enables one to use more simple expressions for studying such important parameters as layer thicknesses and strains. Third, the fact that contribution from a layer into reflectivity does not depend on the layer structural perfection and makes it possible to separate information from another structure layers . 
V.P. Kladko et al.: Investigation of superlattice structure ...

\section{References}

1. V. Holy, U. Pietch, T. Baumbach, High-Resolution X-Ray Scattering from Thin Films and Multilayers. - Berlin, Springer. - 1998. - $254 \mathrm{p}$.

2. Tapfer I., Ploog K. X-ray interference in ultrathin epitaxial layers: a versatile method for the structural analysis of single quantum wells and heterointerfaces // Phys. Rev. B., 40(14), pp. 9802-9810 (1989).

3. V.S. Speriosu, T. Vreelang, X-ray rocking curve analysis of superlattice. // J. Appl. Phys., 56(6), pp. 1591-1600 (1984).

4. V.P. Klad'ko, L.I. Datsenko, V.F. Machulin, V.B. Molodkin, Satellite intensity behaviour in short-period GaAs/AlAs superlattices at different degrees of layer structure perfec- tion // Metallofiz. i Nov. Tekhnol., 25(4), pp. 556-564 (2003), (in Russian).

5. L.I. Datsenko, V.P. Klad'ko, V.F. Machulin, V.B. Molodkin, $X$-ray Dynamical Scattering by Real Crystals in the Anomalous Dispersion Region. - Kiev, Akademperiodika. - 2002. - 352 pp. (in Russian).

6. A.T. Macranger, G.P. Schwartz, G.J. Gualtieri, X-ray and Raman characterization of $\mathrm{AlSb} / \mathrm{GaSb}$ strained layer superlattices and quasiperiodic Fibonacci lattices // J. Appl. Phys., 64(12), pp. 6733-6745 (1988).

7. M. Haase, W. Prost, P. Velling, Q. Liu, F.J. Tegude, HR XRD for the analysis of ultrathin centrosymmetric strained DBRTD heterostructures // Thin Sol. Films., 319(1-2), pp. 25-28 (1998). 